\title{
Induced spatiotemporal modulation instability in a noninstantaneous self-defocusing medium
}

\author{
Wen-Han Chu, Chien-Chung Jeng, Chao-Hsian Chen, Yi-Hsin Liu, and Ming-Feng Shih \\ Department of Physics, National Taiwan University, Taipei 106, Taiwan
}

Received December 14, 2004

We demonstrate theoretically and experimentally that induced spatiotemporal modulation instability can exist in a self-defocusing medium if the nonlinearity is noninstantaneous. We predict the growth rate as a function of the spatial and temporal frequencies of the modulation and the response time of the nonlinearity and confirm it by our experiments. (C) 2005 Optical Society of America

OCIS codes: $190.3100,190.4420,190.5330$.

Modulation instability (MI), which happens in many nonlinear wave systems, ${ }^{1-3}$ is when a small amplitude modulation of a carrier wave grows exponentially due to the nonlinear response of the medium. To form MI, the Lighthill criterion ${ }^{3}$ should be satisfied; that is, the nonlinearity and the dispersion (diffraction) must work oppositely. For example, optical temporal MI can happen in a fiber when it is selffocusing and of anomalous dispersion. ${ }^{2}$ Also, in selffocusing media, a cw light beam can form spatial $\mathrm{MI}^{3-6}$ unless the light beam is incoherent enough and propagates in a noninstantaneous nonlinear medium. ${ }^{7,8}$ Oppositely, it is thought that MI cannot normally form in a self-defocusing medium because the nonlinearity and the diffraction work in the same way. The only exceptions are when the defocusing medium is within a cavity ${ }^{9}$ or when there are two counterpropagating beams in the defocusing medium. ${ }^{10}$

Nevertheless, the stability of a system is often affected by its delayed response. For example, in selffocusing media, the stability of partially incoherent solitons, ${ }^{11}$ stimulated four-photon interactions, ${ }^{12}$ or the spatial $\mathrm{MI}^{13}$ could be altered by the noninstantaneity of the nonlinear response. In this Letter, we demonstrate both theoretically and experimentally that the induced spatiotemporal MI indeed can happen in a noninstantaneous self-defocusing medium.

We first analyze the wave propagating in the nonlinear medium, starting from $\nabla^{2} E-\mu\left(\partial^{2} D / \partial t^{2}\right)=0$, with $D=\varepsilon\left(|E|^{2}\right) E$ being the displacement. This equation is rewritten as

$$
\nabla^{2} E=\mu \frac{\partial^{2}(\varepsilon E)}{\partial t^{2}}=\mu \varepsilon \frac{\partial^{2} E}{\partial t^{2}}+2 \mu\left(\frac{\partial \varepsilon}{\partial t}\right)\left(\frac{\partial E}{\partial t}\right)+\mu\left(\frac{\partial^{2} \varepsilon}{\partial t^{2}}\right) E .
$$

Assuming that $\varepsilon=\varepsilon_{0}\left(n_{0}+\delta n\right)^{2}$ and the nonlinearity is of the relaxation type with time constant $\tau$, we have $\delta n\left[|E(t)|^{2}\right]=\int_{-\infty}^{t}(1 / \tau) F\left[\left|E\left(t_{1}\right)\right|^{2}\right] \exp \left[-\left(t-t_{1}\right) / \tau\right] \mathrm{d} t_{1}$, with $F$ being the nonlinearity form. For $\tau$ much larger than the optical period of about $10^{-15} \mathrm{~s},{ }^{14}$ we can neglect the last two terms of Eq. (1). Putting $E$ $=A(\bar{r}, t) \exp (i \omega t-i k z)$ into Eq. (1); neglecting all time derivatives of the envelope $A$, which are much smaller than the second time derivative of $\exp (i \omega t-i k z)$; and using the paraxial approximation, we obtain

$$
\nabla_{\perp}^{2} A-2 i k \frac{\partial A}{\partial z}+2 k^{2} \frac{\delta n\left(|A|^{2}\right)}{n_{0}} A=0 .
$$

To study MI, we let $A=\left(A_{0}+a\right) \exp (-i \gamma z)$ as the unperturbed plane wave plus a small modulation, ${ }^{13}$ where $\gamma=k F\left(A_{0}^{2}\right) / n_{0}$ and $a=a_{1}+i a_{2}$ is the small modulation with $a_{1,2}=\operatorname{Re}\left[a_{1,2}^{0} \exp \left(i \Omega \cdot t+i \vec{k}_{\perp} \cdot \vec{r}-i h_{1} z+h_{2} z\right)\right]$. Here, $h_{1}$ and $h_{2}$ are the modified propagation constant and the exponential growth rate, respectively. This is different from the instantaneous case, in which either $h_{1}$ or $h_{2}$ will be zero. Since $\delta n\left(\left|A_{0}+a\right|^{2}\right)$ $\approx F\left(A_{0}^{2}\right)+\operatorname{Re}\left[2 A_{0} \kappa a_{1}^{0} \exp \left(i \Omega \cdot t+i \vec{k}_{\perp} \cdot \vec{r}-i h_{1} z+h_{2} z\right) /(1\right.$ $+i \Omega \tau)]$, with $\kappa=-[\mathrm{d} F(I) / \mathrm{d} I]_{I=A_{0}^{2}}>0$ in a defocusing medium, and with the integration being carried out, Eq. (2) becomes

$$
\begin{gathered}
\nabla_{\perp}^{2}\left(a_{1}+i a_{2}\right)-2 i k \frac{\partial\left(a_{1}+i a_{2}\right)}{\partial z}-4 k^{2} \kappa \operatorname{Re}\left[\frac{A_{0}^{2} a_{1}^{0}}{n_{0}(1+i \Omega \tau)}\right. \\
\left.\times \exp \left(i \Omega \cdot t+i \vec{k}_{\perp} \cdot \vec{r}-i h_{1} z+h_{2} z\right)\right]=0 .
\end{gathered}
$$

We separate the real and imaginary parts and obtain the conditions for which Eq. (3) has nontrivial solutions:

$$
\begin{aligned}
h_{2}= & \pm \frac{\kappa k A_{0}^{2}}{n_{0} \sqrt{2}}\left\{-P^{4}-\frac{2 P^{2}}{1+(\Omega \tau)^{2}}\right. \\
& \left.+P^{2}\left[P^{4}+\frac{4+4 P^{2}}{1+(\Omega \tau)^{2}}\right]^{1 / 2}\right\}^{1 / 2},
\end{aligned}
$$

which is shown in Fig. 1 , and $h_{1}=\left(\kappa k_{\perp}^{2} A_{0}^{2} \Omega \tau\right) / 2\left[n_{0}(1\right.$ $\left.\left.+\Omega^{2} \tau^{2}\right) h_{2}\right]$. Here $P=k_{\perp} /\left[\left(2 \kappa / n_{0}\right)^{1 / 2} \cdot k A_{0}\right]$. Why the spatially and temporally periodic small modulation can grow is intuitively understandable. When the light beam with a transversely spatially periodic intensity propagates in the defocusing medium, the region with lower light intensity should show a higher refractive index. However, due to the delayed response of the medium, if the spatial modulation is moving transversely with a proper frequency, the light of 


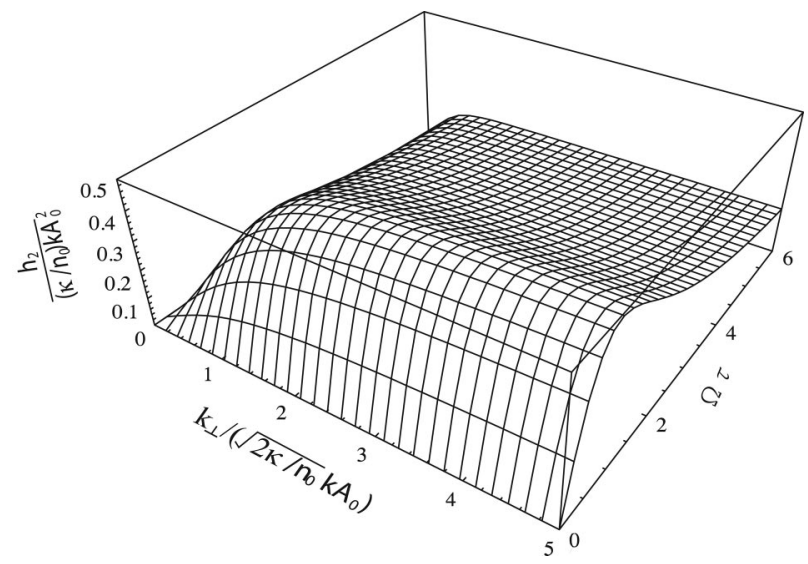

Fig. 1. Gain coefficient of the MI in a noninstantaneous self-defocusing medium.

higher intensity can always meet the higher index, similar to the situation in which MI occurs in an instantaneous self-focusing medium.

Several points deserve more attention. First, if we take the limit $\Omega \tau \rightarrow 0$, then $h_{1} \rightarrow\left[\left(k_{\perp}^{4} / 4 k^{2}\right)\right.$ $\left.+\left(\kappa k_{\perp}^{2} A_{0}^{2} / n_{0}\right)\right]^{1 / 2}$, which is the same as that in an instantaneous defocusing medium, and $h_{2} \rightarrow 0$, meaning no MI exists. Second, if $\Omega \tau$ approaches infinity, then $h_{2}$ approaches zero. In this situation, MI cannot happen since the intensity varies too fast for the material to respond. Third, for a fixed value of $\Omega \tau, h_{2}$ is constant over a range of $k_{\perp}$ and contains no peak. As a result, no spontaneous MI can happen in the noninstantaneous self-defocusing medium. Fourth, though $h_{2}$ is nonzero for infinite spatial frequencies $\left(\vec{k}_{\perp}\right)$, meaning there is an unlimited spatial gain bandwidth, this actually would not happen if the paraxial approximation $\left(\left|\vec{k}_{\perp}\right| \ll k\right)$ is relaxed.

The experimental setup (Fig. 2) is similar to those used in Refs. 4 and 6. A collimated light beam from a $532 \mathrm{~nm} \mathrm{cw}$ laser is separated into two beams, A and B. B's amplitude is less than 1\% of A's. B is recombined with A with a slightly slanted angle to generate the light beam that carries the spatially periodic modulation by interference. The intensity of $A+B$ is about $I_{1}=162 \mathrm{~mW} / \mathrm{cm}^{2}$. We launch $\mathrm{A}+\mathrm{B}$ into a photorefractive SBN:60 crystal, whose length is $7 \mathrm{~mm}$, width along the $c$ axis is $10 \mathrm{~mm}$, and effective electrooptic coefficient is $290 \mathrm{pm} / \mathrm{V}$. The refractive index of the crystal is 2.35 . The image of the light beam at the input face and its numerical Fourier transform are shown in Fig. 3(a). The clear pattern shown is due to the striation of the crystal, whose spatial frequency is $k_{0}$. We notice a small peak at $k_{\perp}\left(k_{\perp x}=52 / \mathrm{mm}, k_{\perp y}\right.$ $=-53 / \mathrm{mm}$ ), with modulation depth $1.0 \%$, which corresponds to the interference bands of $\mathrm{A}+\mathrm{B}$. We make this depth small on purpose to prevent the exponential growth from going into saturation. Also, because the small peak at $k_{\perp}$ is only $1.0 \%$ of that at the zero spatial frequency, we cannot use a lens to get a clear Fourier image due to the limited dynamic range of the camera. We then apply a $0.93 \mathrm{kV}$ voltage across the crystal with its polarity along the $c$ axis to yield the noninstantaneous self-defocusing nonlinearity. ${ }^{15-18}$ The maximal index change is about $1.8 \times 10^{-4}$. The response time is of the order of $0.1 \mathrm{~s}$ for our illuminating intensity. We observe that the modulation depth [Fig. 3(b)] of the static striation $\left(k_{0}\right)$ is greatly reduced from $10 \%$ to $1.0 \%$. This is expected since a large modulation will decay in the selfdefocusing medium unless the modulation is time varying.

We attach a piezo transducer to a mirror in B's optical path. This transducer is driven by an amplified sawtooth signal from the function generator, as shown in Fig. 2. This causes B's optical path to vary and makes the interference bands move 20 periods in a cycle from $\mathrm{t} 1$ to $\mathrm{t} 2$. The temporal frequency $(f$ $=\Omega / 2 \pi)$ of the band shift is adjusted by the slope of the ramp voltage. We then use the CCD camera to take the snapshot images at the output face of the crystal every $0.1 \mathrm{~s}$. Figures 3(c) and 3(d) show two of the snapshot images for a different frequency $f$. From the series of snapshot images, we find that the bands corresponding to $k_{\perp}$ move at the same speed and in the same direction as that at the input face, meaning that they are indeed the pattern of induced MI. To obtain the modulation depth and the growth rate, we take the Fourier transform of the snapshot images. For each frequency $f$ we find that the modulation depths are about the same for the last few periods in a cycle, indicating that the system has reached a steady state before t2. We therefore do not need to worry about the phase discontinuity between two cycles. Also, to distinguish our experiment from the well-known two-wave mixing with a moving grating, ${ }^{19}$ in a separate experiment, we reverse the sawtooth signal that drives the mirror to make the bands move in the opposite direction. For the same frequency $f$, we observe about the same modulation

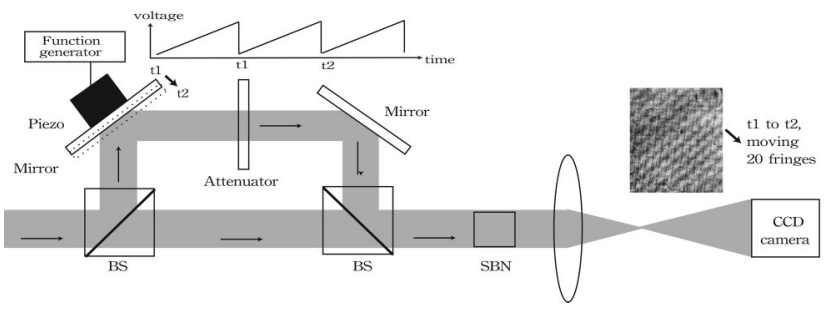

Fig. 2. Sketch of the experimental setup. BS, beam splitter.

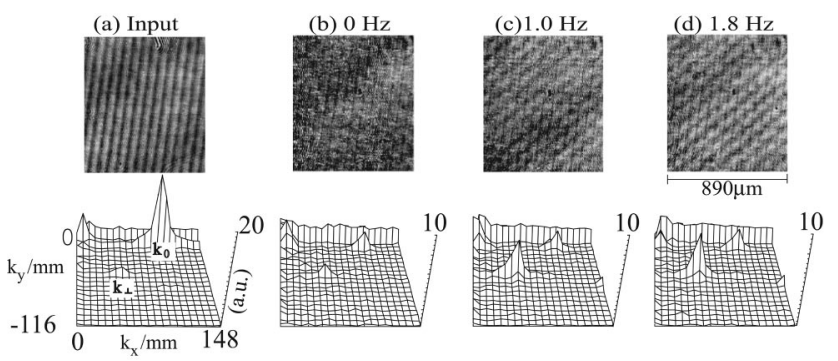

Fig. 3. Intensity patterns at the (a) input and (b)-(d) output faces of the crystal and their Fourier transform. The component at zero spatial frequency is removed manually since it is too high to be shown. 
depths for both directions. This is saying that the gain we observe here is symmetric about the detuning frequency, clearly different from the asymmetric gain of the two-wave mixing.

We want to compare the experimental result with the theoretical prediction. We use the data from the Fourier transform to find the gain, defined as (output modulation depth)/(input modulation depth), and calculate the growth rate, defined as $\ln$ (gain)/(length of the crystal), which is shown as the dots in Fig. 4(b). We use $P_{1}=0.21\left(k_{\perp}=74 / \mathrm{mm}\right.$, $k=27,754 / \mathrm{mm}, n_{0} \approx 2.35$, and $\left.\Delta n=1.8 \times 10^{-4}\right)$ and $\tau_{1}$ $=0.1 \mathrm{~s}$ as the theoretical parameters to fit the experimental result and plot it in Fig. 4(b). In the lowfrequency range $(<4 \mathrm{~Hz})$, the experimental result and the theoretical prediction fit well. For the higherfrequency range $(>4 \mathrm{~Hz})$, the discrepancy can be attributed to the fact that the fringes move too fast so that the transient effect of the charge redistribution and screening process needs to be corrected in theory. We also do another set of experiments with $k_{\perp}$ $=148 / \mathrm{mm}, \quad V_{\text {bias }}=550 \mathrm{~V}\left(\Delta n=1.1 \times 10^{-4}\right), \quad$ and $\quad I_{2}$ $=46 \mathrm{~mW} / \mathrm{cm}^{2}$, shown in Fig. 4(a). We use fitting parameters $P_{2}=0.55$ and $\tau_{2}=0.35 \mathrm{~s} \quad\left(=\tau_{1} \cdot I_{1} / I_{2}\right.$, the response time is inversely proportional to intensity) in the theory. Figure 4(c) shows good agreement between the theory and the experiment.

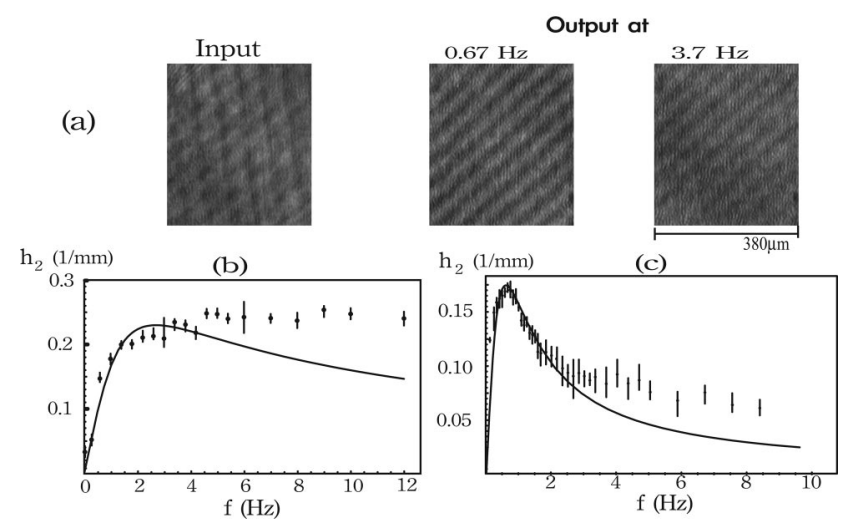

Fig. 4. (a) Intensity patterns of the MI. (b),(c) Growth rates as a function of the temporal frequency of the modulation. The solid curves are predicted by the perturbation.
To conclude, we have demonstrated theoretically and experimentally the induced spatiotemporal modulation instability in a self-defocusing medium when the nonlinearity is noninstantaneous.

We thank the National Science Council, Taiwan, for its support. M.-F. Shih's e-mail address is mfshih@phys.ntu.edu.tw.

\section{References}

1. See, e.g., E. Infeld and G. Rowlands, Nonlinear Waves, Solitons and Chaos (Cambridge U. Press, 2000).

2. G. P. Agrawal, Nonlinear Fiber Optics (Academic, 1995).

3. M. Remoissenet, Waves Called Solitons (SpringerVerlag, 1999).

4. M. Iturbe-Castillo, M. Torres-Cisneros, J. SanchezMondragon, S. Chavez-Cerda, S. Stepanov, V. Vysloukh, and G. Torres-Cisneros, Opt. Lett. 20, 1853 (1995).

5. R. Malendevich, L. Jankovic, G. Stegeman, and J. S. Aitchison, Opt. Lett. 26, 1879 (2001).

6. R. Schiek, H. Fang, R. Malendevich, and G. I. Stegeman, Phys. Rev. Lett. 86, 4528 (2001).

7. M. Soljacic, M. Segev, T. Coskun, D. Christodoulides, and A. Vishwanath, Phys. Rev. Lett. 84, 467 (2000).

8. D. Kip, M. Soljacic, M. Segev, E. Eugenieve, and D. Christodoulides, Science 290, 495 (2000).

9. D. Michaelis, U. Peschel, and F. Lederer, Phys. Rev. A 56, R3366 (1997).

10. W. Firth and C. Pare, Opt. Lett. 13, 1096 (1988).

11. M. Shih and F. Sheu, Phys. Rev. Lett. 86, 2281 (2001).

12. R. Y. Chiao, P. L. Kelley, and E. Garmire, Phys. Rev. Lett. 17, 1158 (1966).

13. M. Shih, C. Jeng, F. Sheu, and C. Lin, Phys. Rev. Lett. 88, 133902 (2002)

14. For most nonlinearities other than that from electronic polarization, this criterion is satisfied; see R. W. Boyd, Nonlinear Optics (Academic, 1992).

15. G. Duree, M. Morin, G. Salamo, M. Segev, B. Crosignani, P. Di Porto, E. Sharp, and A. Yariv, Phys. Rev. Lett. 74, 1978 (1995).

16. Z. Chen, M. Mitchell, M. Shih, M. Segev, M. Garrett, and G. Valley, Opt. Lett. 21, 629 (1996).

17. M. Segev, G. C. Valley, B. Crosignani, P. DiPorto, and A. Yariv, Phys. Rev. Lett. 73, 3211 (1994).

18. M. Segev, M. Shih, and G. Valley, J. Opt. Soc. Am. B 13, 706 (1996).

19. Ph. Refregier, L. Solymar, H. Rajbenbach, and J. Huignard, J. Appl. Phys. 58, 45 (1985). 